\title{
Búsqueda de Tuberculosis en Pacientes Sintomáticos Respiratorios en Cuatro Hospitales de Bogotá D.C.
}

\section{Search for Tuberculosis in patients with the respiratory symptoms in four hospitals of Bogotá D.C.}

\author{
Sandra C. Henao-Riveros ${ }^{1}$, Claudia R. Sierra-Parada², \\ Edgar A. Sánchez-Morales ${ }^{1}$ y Alfredo S. Rodriguez ${ }^{1}$
}

1 Facultad de Medicina, Universidad Nacional de Colombia. schenaor@unal.edu.co, easanchezm@unal.edu.co, asaavedrar@unal.edu.co

2 Universidad Colegio Mayor de Cundinamarca. crsierrap@unal.edu.co

Recibido 5 Diciembre 2006/Enviado para Modificación 19 Junio 2007/ Aceptado 20 Agosto 2007

\section{RESUMEN}

Objetivo Identificar individuos sintomáticos respiratorios que acuden a diferentes senvicios médicos en hospitales de tercer nivel de Bogotá D.C. para el diagnóstico temprano de la Tuberculosis.

Materiales y métodos Se realizó búsqueda activa de sintomáticos respiratorios a través de actividades de promoción y prevención utilizando un formulario para obtención de datos socio demográficos y clínicos. Se investigaron 566 muestras de esputo de 354 individuos sintomáticos respiratorios en cuatro hospitales de Bogotá D.C. Se detecto por baciloscopia y cultivo pacientes con tuberculosis.

Resultados La distribución por género fue de $39 \%$ masculino y $61 \%$ femenino, con un promedio de 57,4 años. El 48,5 \% tenían síntomas respiratorios mayor a dos meses, 53,3 \% presentaban cicatriz BCG, 50 \% pertenecía al estrato 2 y 72,8\% alcanzaba un nivel educativo igual o menor a primaria básica. El porcentaje de captación por baciloscopia fue de 3,67 \% y de baciloscopia y/o cultivo fue del 4,2 \%. Se encontraron 11 casos de Mycobacterium tuberculosis y 2 casos de micobacterias no tuberculosas. La mayoría de pacientes con tuberculosis perteneció al género masculino, presentaban síntomas de tos y expectoración mayor a dos meses y el $54,5 \%$ tenían vacunación BCG.

Conclusiones La búsqueda activa de sintomáticos respiratorios detecta casos de pacientes bacilíferos, fuente de contaminación entre la población, facilita el inicio de la terapia antituberculosa aplicando la estrategia DOTS. El éxito en el control de la tuberculosis depende de políticas gubernamentales que sigan las recomendaciones de la OMS.

Palabras Clave: Tuberculosis, hospitales, Bogotá(fuente: DeCS, BIREME) 


\section{ABSTRACT}

Objective To identify individuals with the respiratory symptoms who receive different medical services in third level hospitals of Bogotá D.C. for early diagnosis of Tuberculosis.

Materials and methods An active search for individuals with Tuberculosis respiratory symptoms through promotion and prevention activities was realized utilizing a format for obtaining social, demographic and clinical data. 566 samples of sputum from 354 individuals with respiratory symptoms were investigated in four Bogota hospitals. By means of positive smear and bacillus culture the tuberculosis patients were detected.

Results The gender distribution was $39 \%$ male and $61 \%$ female, with an average age of 57,4 years. $48,5 \%$ had respiratory symptoms for more than two months, 53,3 $\%$ presented BCG scar, $50 \%$ belonged to socioeconomic level 2 and $72,8 \%$ had an education level equal to or less than basic elementary school. The percentage detected by positive smear was of 3,67 \% and by positive smear and/or culture was of 4,2\%. 11 cases of Mycobacterium tuberculosis and 2 cases of non tuberculosis mycobacterium were found. The majority of patients with tuberculosis belonged to the masculine gender, presented coughing symptoms and expectoration greater than two months and 54,5\% had BCG vaccination.

Conclusions The active search patients with the respiratory symptoms detected cases of patients with positive smear, a source of contamination among the population, which facilitates the anti-tuberculosis therapy applying the DOTS strategy. The success of tuberculosis control will depend on government policies that follow the OMS recommendations.

Key Words: Tuberculosis, symptoms, hospitals, Bogotá (source: MeSH, NLM).

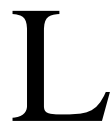

a tuberculosis (TBC) representa una alta tasa de morbi-mortalidad en el mundo, asociada a factores como las malas condiciones socioeconómicas, sanitarias, dificultades en la promoción y prevención de la salud, poco compromiso gubernamental y patologías como la diabetes, cáncer, terapias inmunosupresoras y la coinfección con el VIH (1-3).

La Organización Mundial de la Salud(OMS) declaró la Tuberculosis como una emergencia global en 1993, y la estrategia eficaz para su control sigue siendo la interrupción de la transmisión mediante la detección activa de pacientes sintomáticos respiratorios y la cura rápida de los casos infecciosos; la baciloscopia de esputo como herramienta diagnóstica para la detección del bacilo tiene alta especificidad, aporta el diagnóstico primario de una manera rápida, es de bajo costo y se puede implementar en cualquier lugar (4- 6). 
El éxito del control de la tuberculosis se basa en la implementación de la estrategia DOTS (Tratamiento Directamente Observado), esta estrategia comprende cinco principios básicos: el compromiso político para el control de la tuberculosis, el diagnóstico bacteriológico de calidad y accesible a la población, la dotación permanente de medicamentos de calidad certificada, el tratamiento con esquemas acortados estandarizados y toma de medicación directamente observada y el sistema de registro e información para el monitoreo y evaluación de las intervenciones $(2,7,8)$.

En Colombia, los reportes oficiales del programa de Control de la tuberculosis muestran una disminución de los casos nuevos, siendo esto un reflejo de las pocas acciones de búsqueda, el detrimento de los Programas, la deficiencia en los sistemas de registro y de la no sospecha por parte del clínico de un caso de tuberculosis $(9,10)$.

El objetivo de este estudio fue identificar individuos sintomáticos respiratorios que acuden a cuatro hospitales de tercer nivel de Bogotá para el Diagnóstico Temprano de tuberculosis.

\section{MATERIALES Y METODOS}

Se realizaron actividades de promoción y prevención mediante educación (charlas, conferencias y plegables) a individuos adultos de ambos sexos que asistieron a los servicios médicos por diferentes motivos de consulta en cuatro instituciones de salud en la ciudad de Bogotá D.C. (Hospital San Blas, Hospital El Tunal, Hospital Simón Bolívar y Clínica San Pedro Claver). Las actividades incluyeron instrucción sobre aspectos sobresalientes de la tuberculosis, su impacto en el individuo y en la comunidad, los síntomas y signos y las medidas de prevención y control de la enfermedad.

Identificación del Sintomático Respiratorio (S.R): se seleccionaron aquellos individuos con tos y/o expectoración mayor a 15 días de evolución que voluntariamente participaran y cumplieran los criterios de Sintomático Respiratorio (5,11-13). Cada individuo luego, del consentimiento informado, fue entrevistado usando un cuestionario estandarizado que incluyó datos sociodemográficos como edad, género, estrato socioeconómico y nivel educativo; datos clínicos como la presencia de tos, expectoración y tiempo de los síntomas y epidemiológicos teniendo en cuenta numero de convivientes, si antes fue diagnosticado como paciente tuberculoso, si era contacto de paciente tuberculoso y la presencia o no de cicatriz BCG. 
Recolección y procesamiento de muestras de esputo: La muestra de esputo fue recolectada por expectoración espontánea al momento del cuestionario, la segunda muestra a la mañana siguiente y la tercera a la entrega de la segunda. Estas fueron sometidas a un proceso de decontaminación con posterior centrifugación a 5.800xg para realizar la baciloscopia por medio de la coloración de Ziehl Neelsen y cultivo por el método de Ogawa modificado por Kudoh $(14,15)$.

Identificación de los cultivos: Los cultivos positivos para la coloración de Ziehl Neelsen fueron identificados por medio de técnicas bioquímicas $(14,16,17)$.

Análisis de datos: Los resultados fueron consignados en una base de datos y analizados por medio del programa estadístico SAS versión 9, para elaborar las frecuencias de las variables sociodemográficas, clínicas y epidemiológicas.

Tipo de estudio: Tamización de oportunidad.

\section{RESULTADOS}

Se investigaron un total de 566 muestras de esputo provenientes de 354 individuos el 42,7 \% pertenecían al hospital San Blas, 18,4 \% al Hospital el Tunal, 20,3 \% al Hospital Simón Bolívar y 18,6 \% a la Clínica San Pedro Claver de Bogotá D.C. El $39 \%$ fueron hombres y $61 \%$ mujeres. Las edades oscilaron entre 18 y 93 años con una media para los hombres de 57,9 años y para las mujeres de 56,9 años (Tabla 1).

Tabla 1. Población de sintomáticos respiratorios

\begin{tabular}{lccccc}
\hline \multicolumn{1}{c}{ Institución } & $\begin{array}{c}\text { Pacientes } \\
\text { sintomáticos }\end{array}$ & \multicolumn{2}{c}{ Hombres } & \multicolumn{2}{c}{ Mujeres } \\
& respiratorios & $\mathrm{n}$ & $(\%)$ & $\mathrm{n}$ & $(\%)$ \\
\hline H. San Blas & 151 & 63 & $(41,7)$ & 88 & $(58,3)$ \\
H. Tunal & 65 & 19 & $(29,0)$ & 46 & $(71,0)$ \\
H. Simón & & & & & \\
Bolivar & 72 & 22 & $(30,5)$ & 50 & $(69,5)$ \\
C. San Pedro & 66 & 34 & $(51,5)$ & 32 & $(48,5)$ \\
\hline TOTAL & 354 & 138 & $(39,0)$ & 216 & $(61,0)$ \\
\hline
\end{tabular}

La mayoría de los individuos seleccionados como sintomáticos respiratorios, el 48,5 \% (172 de 354) presentaban síntomas de tos y/o expectoración por un tiempo mayor a dos meses. La presencia de cicatriz de BCG se encontró en el 53,3 \% (189 de 354) de los pacientes sintomáticos respiratorios estudiados. La población estudiada se distribuyo entre los estratos de 1 a 4, encontrándose un 50 \% (177 de 354) en el estrato 2. Respecto al nivel educativo, más de la mitad de los sintomaticos respiratorios incluidos en el estudio 59,6 \% (211 de 354) habían cursado solamente primaria, 22 \% (78 de 354) secundaria, 2,6 \% (9 de 
354) técnico, 2,6 \% (9 de 354) universitario y un 13,2 \% (47 de 354) no tenían ningún nivel educativo.

Frecuencia de Tuberculosis y/o Micobacteriosis

Detección por baciloscopia. Se realizó un total 566 baciloscopias para un promedio de 1,6 por paciente. El cumplimiento en la recolección de la segunda muestra de esputo fue de 41,8 \% (148 muestras) y para la tercera muestra fue del 18,0 \% (64 muestras). El porcentaje de captación de enfermos tuberculosos por baciloscopia fue de 3,7\%, es decir 13 de 354 sintomaticos respiratorios investigados; de los cuales el 30,7 \% (4 de 13) provenían del Hospital San Blas, con un porcentaje de captación para la institución de 2,6 \%, (4 de 151), detectados a la primera baciloscopia. El 38,5 \% (5 de 13) pertenecían al Hospital Simón Bolívar, con un porcentaje de captación en el hospital de 6,9 \% (5 de 72), detectando dos casos en la primera baciloscopia y 3 en la tercera baciloscopia. En el Hospital el Tunal se detecto un caso (7,7 \%) a la primera baciloscopia para un porcentaje de captación para este hospital de 1,4 \%. Un 23 \% (3 de 13) provenían de la Clínica San Pedro Claver mostrando un porcentaje de captación de 4,5 \% (3 de 66), detectando dos casos en la primera baciloscopia y un caso en la tercera baciloscopia (Tabla 2).

Tabla 2. Diagnóstico por baciloscopia

\begin{tabular}{|c|c|c|c|c|c|c|}
\hline \multirow[t]{2}{*}{ Institución } & \multirow{2}{*}{$\begin{array}{c}\text { No. } \\
\text { Pacientes }\end{array}$} & \multirow{2}{*}{$\begin{array}{l}\text { No. } \\
\text { Muestras }\end{array}$} & \multirow{2}{*}{$\begin{array}{c}\text { Pacientes } \\
\text { positivos a la } \\
\text { Baciloscopia }\end{array}$} & \multicolumn{3}{|c|}{ Baciloscopias positivas } \\
\hline & & & & $1^{\mathrm{a}}$ & $2^{a}$ & $3^{a}$ \\
\hline H. San Blas & 151 & 329 & 4 & 4 & 0 & 0 \\
\hline $\begin{array}{l}\text { H. Simón } \\
\text { Bolivar }\end{array}$ & 72 & 118 & 5 & 2 & 0 & 3 \\
\hline H. Tunal & 65 & 102 & 1 & 1 & 0 & 0 \\
\hline $\begin{array}{l}\text { C. San Pedro } \\
\text { Claver }\end{array}$ & 66 & 107 & 3 & 2 & 0 & 1 \\
\hline TOTAL & 354 & 566 & 13 & $9(69,2 \%)$ & 0 & $4(30,8 \%)$ \\
\hline
\end{tabular}

Detección por cultivo: Al cultivo fueron positivos 13 pacientes para un porcentaje de captación por cultivo de 3,67 \%. En los Hospitales El Tunal y San Blas se diagnosticó un caso por cultivo con baciloscopia negativa para cada uno.

Dos de los cinco casos detectados por baciloscopia en el Hospital Simón Bolívar, no presentaron crecimiento al cultivo. En la Clínica San Pedro Claver los pacientes con baciloscopia positiva presentaron cultivo positivo. 
El porcentaje total de captación por baciloscopia y/o cultivo entre pacientes sintomaticos respiratorios ingresados al estudio fue de 4,2\%. De los 13 aislamientos positivos 11 (84,6 \%) pertenecían al complejo Mycobacterium tuberculosis y $2(15,4 \%)$ a Micobacterias no tuberculosas (MNT), muestras que provenían del Hospital Simón Bolívar y correspondieron a dos pacientes VIH positivos. La confirmación de la presencia de especies pertenecientes al complejo M. tuberculosis fue realizada por medio de pruebas bioquímicas como la detección de Niacina, reducción de nitratos y actividad de catalasa a temperatura ambiente y $68^{\circ} \mathrm{C}$ (Tabla 3).

\begin{tabular}{lcccc}
\multicolumn{5}{r}{ Tabla 3. Casos positivos por baciloscopia y/o cultivo } \\
\hline Institución & $\begin{array}{c}\text { Pacientes con } \\
\text { Cultivos Positivos }\end{array}$ & $\begin{array}{c}\text { Pacientes con } \\
\text { Cultivo Negativo } \\
\text { y Baciloscopia } \\
\text { Positiva }\end{array}$ & $\begin{array}{c}\text { Total Positivos } \\
\text { Baciloscopia y/o } \\
\text { Cultivo }\end{array}$ \\
\hline H. San Blas & 4 & 1 & & 5 \\
H. Simon Bolivar & 3 & & 2 & 5 \\
H. Tunal & 1 & 1 & & 2 \\
\hline C. San Pedro Claver & 3 & & 2 & 3 \\
\hline TOTAL & 11 & 2 & & 15 \\
\hline
\end{tabular}

Se encontró que el 72,7 \% de los pacientes con tuberculosis pertenecían al género masculino, la edad promedio fue de 51,6 años, el 54,5 \% presentaban síntomas respiratorios con tos y expectoración por mas de dos meses, convivían en promedio con 5,54 personas, 2 de los 11 pacientes habían tenido contacto previo con pacientes tuberculosos, un paciente refirió antecedente de tuberculosis y 6 de los 11 tenían cicatriz BCG (Tabla 4).

\begin{tabular}{ccccccccc}
\multicolumn{7}{c}{ Tabla 4. Descripción de pacientes sintomáticos respiratorios } \\
con diagnóstico de tuberculosis
\end{tabular}


Los resultados tanto positivos como negativos para baciloscopia y/o cultivo fueron entregados a las instituciones participantes en el estudio con el fin de tomar las acciones pertinentes.

\section{DISCUSION}

La búsqueda activa de sintomáticos respiratorios para el diagnóstico de la tuberculosis pulmonar se constituye como una de las herramientas más importantes desde el punto de vista de salud pública. Con la detección de casos en la comunidad se cumple con el principal objetivo de la estrategia del tratamiento directamente observado (DOTS) recomendada internacionalmente por la OMS que intenta la detección de por lo menos el 70 \% de los casos y la cura del $85 \%$ de los casos nuevos con baciloscopia positiva. Sin embargo en el informe del 2006 para el Control Global de la Tuberculosis de la OMS la detección global de casos para el 2004 fue del 53 \% y se calcula que sea de 60 \% para el 2005 lo cual esta por debajo la meta del 70 \%, sin embargo con esperanzas que en la región de Las Américas se cumpla con los objetivos de detección y tratamiento de casos nuevos.

Siendo la tuberculosis una enfermedad que presentó para el año 2004 un total de 8,9 millones de casos nuevos en el mundo, de los cuales 3,9 millones fueron bacilíferos, con un total de 1,7 millones de muertes por TBC, se hace necesario continuar implementando las políticas de detección de casos utilizando las herramientas mas eficaces en términos de diagnostico y eficientes en términos económicos (7).

Aunque en Colombia la implementación del Sistema General de Seguridad Social en Salud (SGSSS) amplió la cobertura $(18,19)$, ésta presenta varios inconvenientes en el Control y Prevención de la Tuberculosis debido a la falta de compromiso gubernamental, la aparición de diferentes prestadores en el sistema de salud, la deficiencia de recursos, las dificultades en el suministro de los medicamentos y los sistemas de registro ineficientes, y aunque existe la normatividad necesaria para el seguimiento y control, la tuberculosis no es realmente una prioridad en Salud Pública (20). Recientemente Colombia aparece como uno de los países de Las Américas donde la estrategia DOTS se ha implementado en el diagnóstico bacteriológico y la terapia acortada supervisada con falta de implementación en todas las áreas del monitoreo y seguimiento (7). 
Es así como los trabajos de Carvajal y cols $(10,19)$ en el Valle del Cauca muestra que la percepción en la implementación de los programas de control de la tuberculosis en el marco del Sistema General de Seguridad Social en Salud presenta serios inconvenientes y requiere de un mayor compromiso gubernamental.

En el presente estudio se realizó la búsqueda activa de pacientes sintomáticos respiratorios que asisten a hospitales de tercer nivel, diferenciándose de la búsqueda pasiva donde solo es a través de la consulta médica que detecta los casos de pacientes sintomáticos respiratorios para realizar la baciloscopia de esputo. Los pacientes examinados fueron en su mayoría pertenecientes a los estratos 1 y 2 cercanos a las áreas de influencia de los hospitales, esta población tenía un nivel educativo inferior o igual a educación básica primaria en un 72,8 $\%$. A pesar de la información y estímulo a pacientes para la obtención de las tres muestras de esputo se logro un promedio de 1,6 muestras por paciente igual a lo reportado en el consolidado del programa de tuberculosis para Colombia (9), lo que sin lugar a duda disminuye aproximadamente un $20 \%$ la posibilidad de lograr una baciloscopia positiva en pacientes bacilíferos $(4,11)$. En nuestro estudio la captación por baciloscopia fue de 3,67 \% al realizar las actividades de búsqueda activa en los diferentes centros hospitalarios dato similar al reportado por Chaparro et al (9) para el año 2002 donde el promedio Nacional de positividad de la baciloscopia fue de 3,8 \%. Según la Guía de Atención Integral (5) la positividad debe estar alrededor de 4 \%. Se encontró que el número de sintomáticos respiratorios examinados para obtener un caso positivo de tuberculosis fue de 27 individuos igual que lo notificado en el año 2002 para el promedio Nacional (9). El rendimiento diagnóstico de la primera baciloscopia fue de $69,2 \%$ y a la tercera baciloscopia el 30,8 \% adicional presentando así diferencias con lo reportado en otros estudios donde en la primera baciloscopia el rendimiento alcanza hasta el $83 \%(4,21)$. Lo anterior refuerza la recomendación de tener al menos 2 baciloscopias en los países de alta prevalencia. Este método es el más eficaz para detectar pacientes tuberculosos y por la especificidad en la detección de bacilos acido alcohol resistentes (BAAR), un resultado positivo esta directamente relacionado con la concentración de bacilos en el esputo. En concentraciones inferiores a 1000 bacilos por mililitro, la probabilidad de obtener un resultado de baciloscopia negativo es cerca del 90 \% y esto podría explicar los casos en los cuales se obtuvo baciloscopia negativa y cultivo positivo que es posible en los pacientes paucibacilares. De manera contraria el cultivo puede detectar cantidades mas pequeñas de bacilos es decir tiene una mayor sensibilidad con respecto a la 
baciloscopia y posibilita la identificación de $M$. tuberculosis y otras especies micobacterianas $(4,16)$.

En dos de los trece aislamientos positivos, se identificaron MNT provenientes de pacientes con VIH, razón por la cual se hace fundamental la utilización del cultivo, estos permiten dirigir la terapia farmacológica y más aún para detectar aislamientos resistentes a los medicamentos antituberculosos de primera línea. Aunque la Guía de Atención Integral obliga a los Prestadores de Salud a realizar cultivo con la tercera muestra de baciloscopia negativa en los casos de alta sospecha, esto no se cumple $(4,5,15,16)$.

En dos pacientes con baciloscopia positiva no hubo crecimiento en el cultivo, ésta situación pudo ser debida a que los pacientes estuviesen bajo terapia antibiótica, caso en el cual los bacilos pueden estar presentes en la baciloscopia pero no ser viables para su desarrollo en el cultivo, por la presencia de Micobacterias con requerimientos de crecimiento especiales para el cultivo, la presencia de Micobacterias no cultivables o por errores en el desarrollo de la técnica $(22,23)$.

Más de mitad de los pacientes diagnosticados con tuberculosis tenían síntomas de tos y expectoración por mas de dos meses lo cual sugiere que los síntomas crónicos pueden estar en relación con tuberculosis pulmonar, sin embargo pone de manifiesto el desconocimiento de la población y de algunos trabajadores de la salud sobre la importancia del sintomático respiratorio y la no sospecha de enfermedad tuberculosa en pacientes tosedores cuando asisten a consultas que no obedecen a enfermedad respiratoria. Adicionalmente las dificultades socioeconómicas y de acceso a los servicios de salud retardan la consulta de estos pacientes. 72,7 \% (8 de 11) de los casos positivos correspondieron al género masculino lo cual esta en relación a que el genero masculino se expone a contactos mas frecuentes en la comunidad $(9,24)$. En promedio los pacientes positivos convivían con 5,5 personas las cuales estaban a riesgo de infección y enfermedad tuberculosa, es por esta razón que se debe insistir en la detección temprana de los casos para iniciar tratamiento y así cortar la cadena de transmisión de la TBC, igualmente en la búsqueda activa de los contactos $(5,25)$. Dos de los pacientes diagnosticados con TBC $(18,1$ $\%)$ referían historia de contacto con paciente tuberculoso (Combe positivo), los casos se ubicaron en los grupos de mayor edad en la comunidad lo cual sugiere una reactivación tuberculosa de una infección adquirida años atrás (5). 
Con respecto a la vacunación con BCG se encontró que el 53,3 \% de la población estudiada presentaba cicatriz BCG lo cual indica una baja cobertura en la población examinada si se tiene en cuenta que en países de alta prevalencia como el nuestro se recomienda la vacunación a edades tempranas. La baja cobertura de la vacunación BCG (54,5 \%) encontrada en los pacientes con tuberculosis en el presente estudio no se asoció con la enfermedad. Esto puede correlacionarse con algunos metanálisis realizados donde se ha encontrado diferentes resultados en la eficacia protectora de la vacunación con valores tan amplios como 0 y 89 \% (26). García et al (27) encontraron en un estudio de prevalencia de sintomáticos respiratorios, infección y enfermedad tuberculosa en Mitú que la cobertura de la vacunación con BCG fue del 94 \% y que la mayoría de la población se encontraba en edades entre uno y cuarenta y cuatro años.

Se concluye que la búsqueda de sintomáticos respiratorios en la comunidad permite detectar a través de la baciloscopia los casos de pacientes bacilíferos que son fuente de contaminación entre la población susceptible, facilita el inicio temprano de la terapia antituberculosa con esquemas de tratamiento adecuados así poniendo en marcha la estrategia DOTS.

Se requiere que las políticas gubernamentales en la implementación del Sistema General de Seguridad Social en Salud adopten de manera estricta y con apoyo financiero y operativo las recomendaciones de la OMS para lograr las metas planeadas con respecto a la detección de casos y el tratamiento de los mismos en todo el territorio nacional.

La aparente disminución en los casos de tuberculosis pulmonar con baciloscopia positiva y el número mayor de tuberculosis extrapulmonares reportadas refleja que han disminuido las acciones de búsqueda de pacientes bacilíferos, es por esto que los sistemas de salud deben de incrementar las acciones de promoción y prevención en los centros de atención tanto de la red privada como pública

Agradecimientos. Este trabajo se realizó gracias a la Financiación obtenida por la División de Investigaciones Bogotá de la Universidad Nacional de Colombia. En los diferentes hospitales al Dr. Carlos Álvarez Hospital Simón Bolívar, Dra. Vilma Giratá Hospital San Blas, Dr. Andrés Torres Clínica San Pedro Claver, Dr. William Aristizabal, Lic. Ruth Ortega Hospital el Tunal. 


\section{REFERENCIAS}

1. ONU. (Internet) Disponible en : http://www.unmilleniumproject.org . Consultado Noviembre del 2006.

2. OPS. OMS. 46 a Sesión del comité Directivo. 57 Sesión del comité Regional. Estrategia Regional para el Control de la Tuberculosis para 2005-2015. Washington D.C., USA, 26-30 de septiembre de 2005.

3. Murcia M, Gómez J, Alvarado F, Bustillo J, Mendivelson E, Gómez B, et al. Frecuency of tuberculosis and non tuberculous mycobacteria in HIV infected patients from Bogotá, Colombia. BMC Infect Dis. 2001; 1:21-27.

4. Toman K. Detección de casos, tratamiento y vigilancia. En: Toman K. Tuberculosis. Segunda edición. Washington, D.C: Organización Panamericana de la Salud; 2006.

5. Ministerio de Salud de Colombia. Guía de atención de la Tuberculosis pulmonar y extrapulmonar. Bogotá D.C., Opciones Graficas Editores; 2002.

6. World Health Organization. TB a global emergency. WHO report on the TB epidemic. WHO/TB/94-177. Geneva;1994.

7. World Health Organization. Global tuberculosis control: surveillance, planning, financing. WHO/HTM/TB/2006.362. Geneva; 2006.

8. Farga V. Hacia la erradicación de la tuberculosis. Rev Chil Enferm Respir. 2006;22:52-54.

9. Chaparro P, García I, Guerrero M, Leon C. Situación dela tuberculosis en Colombia,2002. Biomédica. 2004; 24:102-114.

10. Carvajal R, Cabrera G., Mateus J. Efectos de la Reforma en Salud en las acciones de Control de tuberculosis en el Valle del Cauca, Colombia. Biomédica. 2004; 24(supl):134-148.

11. Machado JE. Estrategia de Búsqueda Activa de Tuberculosis en Pereira. Rev Epidemiol Pereira. 2003; 6:45-52.

12. Secretaria Distrital de Salud de Santa Fe de Bogotá. Prevalencia de Sintomáticos Respiratorios y Tuberculosis pulmonar en Santa Fe de Bogotá - Colombia. Centro de Estudios e Información en Salud. Disponible en: http:// www.saludcapital.gov.co . Consultado Octubre de 2006.

13. English R, Bachmann MO, Bateman ED, Zwarenstein MF, Fairall LR, BheekieA, et al. Diagnostic accuracy of an integrated respiratory guidelines in identifying patients with respiratory symptoms requiring screening for pulmonary tuberculosis: a cross-sectional study. BMC Pulmonary Medicine. 2006, 6:22.

14. Garzón M.C., Naranjo N, Sierra C. R., Llerena C, Orjuela D.L. Bacteriología del Mycobacterium tuberculosis y de micobacterias no tuberculosas. Manual de Procedimientos. Instituto Nacional de Salud. Bogotá;2001. 
15. Murcia M, Gómez J, Alvarado F, Bustillo J, Divels G, Gomez B. et al. Prevalencia de Micobacterias en pacientes VIH/SIDA positivos en Bogotá D.C. Rev. Col. Neumol. 13(4):249-261

16. American Thoracic Society. Diagnostic Standard and Classification of Tuberculosis in Adults and Children. Am J Respir Crit Care Med. 2000;161:1376-1395.

17. Telenti A, Marchesi F, Balz M, BallyF, Bottger EC, BodmerT. et al. Rapid identification of micobacteria to the species level by polymerase chain reaction and restriction enzyme analysis. J. of Clin Microbiology.1993;31(2):175-178.

18. Cespedes J.E, Jaramillo L, Martinez R, Olaya S, Reynales J, Uribe C, et al. Efectos de la Reforma de la Seguridad Social en salud en Colombia sobre la equidad en el acceso yla utilización de servicios en Salud. Rev Salud Pública. 2000; 2(2):145164.

19. Carvajal R, Cabrera G.A, Mosquera J. Percepciones de los efectos de la implementación del Sistema General de Seguridad Social en Salud sobre las acciones de control de Tuberculosis en el Valle del Cauca, Colombia. ColombMed. 2004; 35:179-184.

20. Machado J. E. El control de la Tuberculosis en Pereira en los últimos cinco años. Revista Epidemiológica. Instituto Municipal de Salud de Pereira. 2002; 5(1): 1220.

21. Harries $\mathrm{AD}$, et al. Sputum smears for diagnosis of smear-positive pulmonary tuberculosis. Lancet. 1996; 347:834-835

22. Hanna B. Laboratory Diagnosis. En: Rom WN, Garay SM. Tuberculosis. Philadelphia: Lippincott Williams \&Wilkins; 2004.

23. Solda P.A., Rojo S.C, Cosiansi M.C, Barnes A.I. Frecuencia de tuberculosis pulmonar y extrapulmonar en un Hospital de referencia de la Provincia de Córdoba, 1991 2003. Rev. Argent. Microbiol. 2005;37(2): 89-91.

24. Zuluaga L, Betancur C, Abaunza M, Londoño J. Prevalencia de tuberculosis y enfermedad respiratoria en personas mayores de 15 años dela comuna nororiental deMedellín, Colombia. Bol Of Saint Panam. 1991;111(5):406-412.

25. Ospina S. La tuberculosis, una perspectiva histórico epidemiológica. Infectio. 2001;5 (4): 241-249.

26. Guzmán M, Niederbacher J, Orozco LC, Rodríguez MJ. Cobertura de la BCG en dos poblaciones de Bucaramanga socioeconomicamente diferentes (BCG y clases sociales). Rev. Colomb. Neumol. 2001;13:95-99.

27. García I, De la Hoz F, Reyes, Montoya P, Guerrero MI, León CI.. Prevalencia de sintomáticos respiratorios de infección y enfermedad tuberculosa y factores asociados: estudio basado en población, Mitú, Vaupés, 2001. Biomédica. 2004;24(supl): 124-31. 